\title{
ENSAIO DE RESISTÊNCIA ADESIVA POR MICROTRAÇÃO: REVISÃO DE
} LITERATURA

\section{MICROTENSILE BOND STRENGH TEST: A LITERATURE REVIEW}

\author{
Bruna Uglik Garbui* \\ Cynthia Soares de Azevedo** \\ Adriana Bona Matos***
}

\begin{abstract}
RESUMO
Atualmente, a fim de se avaliar a resistência de união entre substrato e material restaurador, o teste mais utilizado é o ensaio por microtração. Todavia, na literatura encontram-se diversas maneiras de se realizar esse teste, desde a seleção da amostra até a análise dos resultados obtidos. Nesta revisão de literatura serão apresentados os principais métodos para realização do teste de microtração, bem como os fatores que podem afetar nos resultados obtidos por este ensaio.
\end{abstract}

DESCRITORES: Resistência à tração • Esmalte dental • Dentina.

\section{ABSTRACT}

Currently, in order to evaluate the bond strength between the dental substrate and the restorative material, microtensile bond strength is the most used test. The purpose of this review is to describe all of the various modifications of the microtensile bond test in one paper. In the literature there are several ways to perform this test, since the selection of the sample to the analysis of the results. In this literature review will be presented the main methods for microtensile testing, as well as factors that may affect the results obtained by this test. The microtensile test methods offer versatility that cannot be achieved by conventional methods, It is up to the researcher to choose the test characteristics that best suits your needs.

DESCRIPTORS: Tensile strength • Dental enamel • Dentin

\footnotetext{
* Cirugiã-dentista pela Faculdade de Odontologia da Universidade de São Paulo, aluna de Mestrado em Odontologia da Faculdade de Odontologia da Universidade de São Paulo, área de concentração Dentística.

** Cirugiã-dentista pela Faculdade de Odontologia da Universidade de São Paulo, aluna de Mestrado em Odontologia da Faculdade de Odontologia da Universidade de São Paulo, área de concentração Dentística.

*** Cirugiã dentista pela Faculdade de Odontologia da Universidade Federal da Bahia, mestre, doutora e livre docente pela Faculdade de Odontologia da Universidade de São Paulo, área de concentração Dentística. Professora associada do departamento de Dentística da Faculdade de Odontologia da Universidade de São Paulo.
} 
GARBUI BU

AZEVEDO CS

MATOS $A B$

ENSAIO DE RES ISTENNCIA ADESIVA POR MICROTRAÇÃO: REVISÃO DE LITERATURA

REV, ODONTOL, UNIV. CID, SÃO PAULO 2013; 25(1): 47 $57, J A N-A B R$

\section{N T R O D U ÇÃ O}

Através do desenvolvimento dos sistemas adesivos com maior resistência de união, os ensaios de cisalhamento e tração, até então mais comumente utilizados, passaram a apresentar alta incidência de fraturas coesivas de substrato dentinário devido a uma distribuição não uniforme do estresse pela interface adesiva. Por isso, o ensaio de microtração foi desenvolvido por Sano et al. ${ }^{1}$ (1994) e introduzido na Odontologia. Segundo Pashley et al. ${ }^{2}$ (1999), o teste de microtração foi desenvolvido a fim de permitir a avaliação da performance adesiva entre materiais adesivos e pequenas regiões de substrato dental.

Esse teste consiste na modificação da metodologia do teste de tração, na qual houve a diminuição das dimensões dos corpos de prova. Em teste de microtração, um corpo de prova com dimensão padronizada é submetido a um esforço que tende a alongá-lo ou esticá-lo até sua ruptura ${ }^{3}$.

Segundo Pashley et al. ${ }^{2}$ (1999), o teste de microtração apresenta melhor distribuição do estresse na interface de união; menor quantidade de defeitos na interface em comparação com os testes convencionais, o que minimiza a incidência de fraturas coesivas; menor variabilidade - que ocorre de $10-25 \%$ ao invés de 30-35\% nos testes convencionais; maior fidelidade nos resultados, uma vez que em uma área de adesão menor, haverá menor quantidade de defeitos e consequentemente uma medição mais fiel dos valores de tração; e baixo desvio-padrão ${ }^{3}$.

Apesar de ser considerado o teste de adesão mais confiável atualmente, o teste de microtração apresenta diversos parâmetros que podem interferir diretamente nos resultados. Os valores de adesão no teste de microtração em esmalte são geralmente inferiores aos obtidos em dentina ${ }^{4,5}$. No entanto, sabe-se que clinicamente o esmalte apresenta melhores características para adesão em comparação à dentina $^{6,7}$. Tal fato pode ser justificado pelo tipo de adesivo utilizado ou pelo método de obtenção dos corpos de prova ${ }^{8,9}$. Estes, para o ensaio de microtração, são obtidos a partir de sucessivos cortes da amostra com discos diamantados ${ }^{2}$, consequentemente, a fricção entre o disco e o dente restaurado gera vibrações e aquecimento da amostra $^{10}$. Tais fenômenos promovem um grande estresse mecânico nas estruturas envolvidas, principalmente no esmalte, por ser um substrato altamente friável e com alto módulo de elasticidade ${ }^{11}$. Tal estresse pode promover a propagação de trincas e até fratura dos espécimes, mascarando os valores reais de resistência de união.

Outra limitação desse método relaciona-se à padronização da delimitação do grupo amostral. Os corpos de prova fraturados prematuramente durante o procedimento de corte da amostra são incluídos na estatística por alguns autores ${ }^{8,12}$ enquanto que outros os excluem do trabalho ${ }^{13}$. Desse modo, a inclusão ou não dos corpos de prova fraturados prematuramente podem causar resultados mais ou menos representativos no trabalho e possivelmente poderá mascarar os resultados reais.

O objetivo deste trabalho é apresentar os fatores que podem interferir nos resultados obtidos através do teste de microtração.

REVISÃO E DISCUSSÃO DA LITERATURA

\section{Seleção da amostra}

$\mathrm{Na}$ literatura consultada, foi possível observar que existe uma preferência em utilizar molares humanos para esse tipo de ensaio ${ }^{1,4,5,7-9,11-61}$.

Cerca de 10,5\% dos artigos analisados para a realização desta revisão utilizaram dentes bovinos ${ }^{62-70}$. Os incisivos superiores permanentes bovinos podem ser utilizados em testes de resistência de união devido a sua semelhança com dentes humanos $^{26,63,66,67,70}$. Entretanto, deve-se ter cautela ao usar esse tipo de dente já que o esmalte bovino não erupcionado tem uma concentração de carbonato mais elevada que o esmalte humano, indicando que o mesmo seja mais susceptível ao ataque ácido devido às variações na hidroxiapatita $^{32,65}$. Outra diferença em relação ao dente humano que deve ser levada em consideração ao escolher o tipo de dente utilizado na pesquisa é o fato dos túbu- 
los dentinários bovinos serem em maior quantidade e terem maior diâmetro $26,64,66$. Adicionalmente, observa-se que a espessura de substrato disponível na vestibular pode dificultar a confecção dos espécimes.

Independente do tipo de substrato que será analisado, depois de extraídos, em todos os artigos consultados, os dentes foram lavados com água e sabão, raspados com curetas periodontais para remoção de resíduos orgânicos e limpos por profilaxia com pedra pomes e água, realizada com escova Robinson em baixa rotação.

Antigamente, Titley et al. ${ }^{68}(1998)$ sugeriram a utilização de dentes recém-extraídos para resultados ideais em testes mecânicos, uma vez que alterações morfológicas na dentina podem ocorrer após a extração dental. No entanto, sabe-se da dificuldade de se obter dentes recém-extraídos, bem como suas implicações legais. Dessa forma, os Bancos de Dentes Humanos surgem como uma opção, com a desvantagem de não se ter conhecimento da procedência detalhada dos dentes. O pesquisador deve ser capaz de coletar e selecionar os dentes para compor a amostra do seu estudo, estando consciente da procedência, idade do paciente, estado do dente na boca e data da extração, sem perda de atenção quanto aos aspectos éticos envolvidos nesse processo, devendo evitar o uso de dentes com procedência desconhecida para compor a amostra.

\section{Substância para armazenamento da} amostra

Imediatamente após a seleção, sugere-se que os dentes extraídos não fiquem em ambiente seco, a fim de evitar alterações estruturais. As técnicas estudadas para seu armazenamento até o uso vão desde o congelamento ${ }^{69}$, refrigeração $0^{49}$ até o armazenamento em temperatura ambiente. Para tanto, pode-se utilizar substâncias tais como água destilada ${ }^{18,29,49,56,58,59}$ solução fisiológica ${ }^{34}$, timol $^{29,33,50,62,67}$, e cloramina

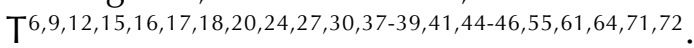
Os protocolos com timol e cloramina $T$ são indicados para evitar crescimento bacteriano, sem alterar a morfologia do substrato ${ }^{33,50,62}$.

O protocolo de armazenamento mais atual para os dentes humanos é a utiliza- ção da cloramina T a 0,5\%, sob refrigera-

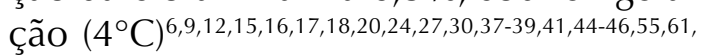
64,71,72, mas também é comum o armazenamento em água destilada $18,29,49,56,58,59$. No entanto, um estudo recente de Mobarak et al. ${ }^{29}$ (2010) concluiu que o tempo e a forma de armazenamento não afetam os resultados obtidos no teste de microtração, ao comparar dentes recém-extraídos, dentes armazenados por 2 anos em cloramina-T a $0,5 \%$ sob refrigeração e dentes secos posteriormente reidratados no momento da execução do experimento. Portanto, um consenso ainda não foi definido pelos pesquisadores.

\section{Seccionamento para obtenção de substrato teste}

Quando o substrato escolhido é a dentina, o método mais usado na literatura consultada consiste na secção do esmalte oclusal no terço médio da coroa dental, perpendicularmente ao seu longo eixo pelo corte de um disco diamantado acoplado a uma máquina de corte de precisão, sob refrigeração à água para expor uma superfície plana de dentina ${ }^{18,22,24,30,31,34,38,39,50,54,58,59}$. Segundo esses artigos, após o corte a superfície da dentina é desgastada, utilizando-se lixas de carbeto de silício de granulação decrescente 120, 240, 320, 400 e 600 com refrigeração abundante à água em politriz acionada em baixa velocidade (100 RPM).

A maioria dos autores $18,22,34,38,39,40,58,59$ utilizam a lixa de granulação \#600 para criar uma camada de esfregaço padronizada $^{53}$. O tempo de uso dessa lixa varia entre 10 segundos $^{34}$ e 60 segundos $^{22,59}$ sendo que o último tempo é o mais utilizado.

Para impedir que os grãos das primeiras lixas interfiram na qualidade do polimento das seguintes, entre cada etapa de polimento os espécimes são levados a um aparelho de ultrassom, com água deionizada com imersão limitada a 5 minutos ${ }^{73}$.

Outro método para remoção do esmalte oclusal é feito através do uso de instrumentos cortantes rotatórios, tais como brocas carbide ${ }^{46,74}$ ou pontas diamantadas $^{45-47,74}$. Porém, é necessário ressaltar que cada método de preparo do substrato produz uma camada de smear diferente, o que afeta diretamente na resistência de
GARBUI BU AzEVEDO CS MATOS $A B$

ENSAIO DE RESISTENNCIA ADESIVA POR MICROTRAÇÃO: REVISÃo de LITERATURA
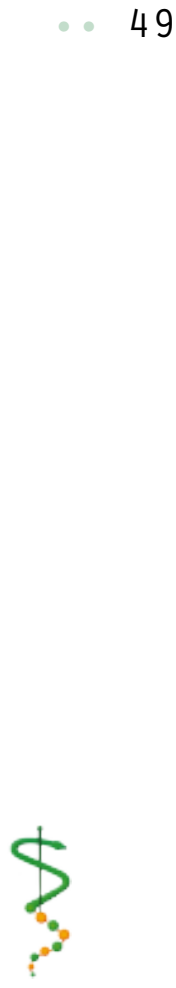

REV. ODONTOL. UNIV. CID. SÃO PAULO 2013; 25(1): 47 57, JAN-ABR 
GARBUI BU

AZEVEDO CS

MATOS $A B$

ENSAIO DE RES ISTÊNCIA ADESIVA POR MICROTRAÇÃO: REVISÃO DE LITERATURA

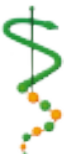

REV, ODONTOL UNIV, CID, SÃO PAULO $2013 ; 25(1): 47-$ 57, JAN-ABR união, principalmente quando o sistema adesivo aplicado é do tipo autocondicionante $\mathrm{e}^{63,46,74}$.

Quando o preparo é feito com brocas carbide essa camada é mais fina se comparada às pontas diamantadas ou às lixas de $\mathrm{SiC}^{46,74}$, isso porque as brocas carbide cortam a superfície ao invés de desgastá-la ${ }^{55}$. Todavia, no estudo de Sattabanasuk et al. ${ }^{46}$ (2007), foi observado que, quando o preparo do substrato era feito com brocas carbide, mesmo tendo uma camada de esfregaço mais fina, a resistência de união era menor.

Já, quando o substrato escolhido é o esmalte, as regiões comumente preparadas são as faces livres ou as proximais dos dentes ${ }^{4,5,9,20,44,52,56,61,63,67}$. Para o desgaste da face, usam-se somente lixas de granulação decrescente. Além disso, é recomendado que após o aplainamento da superfície seja usado um microscópio estereoscópico a fim de se verificar se há somente a presença de esmalte ${ }^{4,5,9,20,44,52,56,61,63,67}$.

Construção dos corpos de prova

Para a construção dos corpos de prova, cada autor utilizou o sistema adesivo escolhido de acordo com as instruções do fabricante. A montagem da camada de resina composta em todos os estudos consultados foi feita em incrementos polimerizados individualmente de acordo com o tempo indicado pelo fabricante, a fim de reduzir a contração de polimerização. Na literatura é mais comum a construção de incrementos com $1 \mathrm{~mm}$ cada $^{22,34,38,41,42,45,46,58,59}$. Porém, o tamanho do bloco de resina composta varia de $2 \mathrm{~mm}^{24}$ a $6 \mathrm{~mm}^{40}$, sendo que o mais comum possui $4 \mathrm{~mm}$ de altura $34,42,46,58,59$.

Após a confecção do bloco de resina, os dentes devem ser armazenados em água destilada por $24 \mathrm{~h}$ em estufa a $37^{\circ} \mathrm{C}^{1,4,5,7-9,12-61}$.

Recomenda-se que tanto a adesão quanto os incrementos de resina devem ser realizados pelo mesmo operador a fim de padronizar a confecção dos corpos de prova.

Quando a superfície analisada é o esmalte, a camada de resina composta deve ser confeccionada somente sobre a área previamente delimitada $4,5,9,20,44,52,56,61,63,67$. Já na dentina, a resina deve ser feita so- bre toda a superfície dentinária, podendo-se deixar uma parte do esmalte aparente a fim de facilitar a localização do limite amelodentinário durante o corte dos pali$\operatorname{tos}^{19,22,30,31,33,38,39,45-47,58,59,74}$

\section{Formato dos corpos de prova:}

$\mathrm{Na}$ literatura consultada para esta revisão, foram encontrados três tipos de formado de corpo de prova: ampulheta, palito e halteres.

\section{Ampulheta:}

Foi o primeiro formato de corpos de prova, proposto por Sano et. al. ${ }^{1} 1994$. Recomendava-se o uso de espécimes em dentina na forma de ampulheta com áreas de união entre 1,6 e 1,8mm² em secções retangulares, pois estas promoviam menor variabilidade e maior porcentagem de fratura adesiva. Sano et al.' (1994) perceberam também que há uma relação inversamente proporcional entre a área de união do corpo de prova e a resistência de união, ou seja, quanto maior a área de união menor será a resistência de união.

Devido a isso, Phrukkanon, Burrow e Tays $^{35,36}$ (1998a.b) propuseram corpos de prova com área de secção transversal de $1,5 \mathrm{~mm}^{2}$ que, além de serem fáceis de confeccionar e testar, apresentam ocorrência mínima de fraturas prematuras durante sua obtenção. Esses autores verificaram também que as secções retangulares, como as propostas por Sano et al. ${ }^{1}$ (1994), poderiam criar a distribuição não uniforme do estresse pela interface de união, influenciando os valores de resistência de união obtidos.

Shono et al. ${ }^{52}$ (1997) testaram corpos de prova na forma de ampulheta em esmalte e comprovaram que, assim como na dentina, no esmalte há uma relação inversa entre a resistência de união e a área. Esses autores recomendaram também que a área de adesão no esmalte para microtração fosse de $1 \mathrm{~mm}^{2}$, já que áreas menores, além de dificultarem as técnicas de confecção da amostra, apresentam grande variabilidade de resultados.

Para criar corpos de prova na forma de ampulheta, geralmente usam-se pontas diamantadas em alta rotação com refrigeração, sem muita pressão a fim de evitar fraturas prematuras ${ }^{71}$. Todavia, mesmo com esses cuidados, o índice de fraturas 
prematuras é alto, tanto em dentina quanto em esmalte, principalmente em áreas reduzidas $^{35,36}$. Além disso, a geometria do pescoço da ampulheta deve ser cuidadosamente analisada, uma vez que o raio de curvatura do entalhe tem influência significativa sobre os valores de concentração de tensões ${ }^{28}$.

\section{Palito:}

Forma proposta por Shono et al. ${ }^{51}$ (1999), em cortes seriados de $1 \mathrm{~mm}$ de espessura, devido à alta incidência de fraturas prematuras e a uma grande dispersão nos resultados de resistência de união obtidos entre os diversos corpos de prova em forma de ampulheta provenientes de um mesmo dente.

Esse tipo de formato é o mais utilizado atualmente $9,13,14,18,20,22,23,30,31,37-39,41,42,45-47$, $49,58,59,61,62,67$.

O formato em palito proporciona um maior número de corpos de prova por dente (20-30 palitos, ao invés de 4-6 ampulhetas), elimina o passo crítico da confecção do entalhe, induz menor estresse na interface de união gerando, então, redução de fraturas prematuras e pode ser usado tanto em esmalte ${ }^{5,22,60}$ quanto em dentina ${ }^{8,15,22}$.

Devido a algumas dificuldades referentes à obtenção de palitos com disco diamantado em esmalte (substrato extremamente friável), foi desenvolvido um fio impregnado por diamante capaz também de realizar os cortes, com menor potencial de danificar o espécime (criação de defeitos, trincas e estresse adicional). Assim, a utilização de fio impregnado por diamante é uma boa alternativa para obtenção de palitos em esmalte ${ }^{9}$.

\section{Halteres:}

O formato de halteres foi desenvolvido uma vez que este mostrou em testes uma distribuição homogênea de estresse na região da interface adesiva ${ }^{43}$. No entanto, para tal característica, é necessária a utilização de um equipamento especialmente concebido para a confecção dos entalhes padronizados com uma geometria mais uniforme, havendo consequentemente uma menor geração de tensões na região, quando comparado com a confecção puramente manual ${ }^{27}$.

Ensaio de resistência adesiva
Inicialmente acreditava-se que a velocidade de tração de um teste mecânico poderia influenciar nos valores de resistência de união. No entanto, estudos demonstraram que não há diferença nos resultados com a velocidade de carga na faixa de 0,01 a $1 \mathrm{~mm} / \mathrm{min}^{25,40,44,57}$.

A velocidade de $1 \mathrm{~mm} / \mathrm{min}$ é a mais utilizada ultimamente $9,18,22,33,34,40-42,45-47,50,58,59,61$, pois resultados demonstraram um padrão de distribuição de estresse mais unifor$\mathrm{me}^{75}$. Há estudos que também utilizam a velocidade de $0,5 \mathrm{~mm} / \mathrm{min}$ para execução

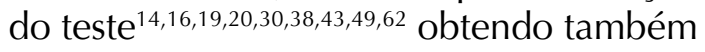
resultados positivos. Dessa forma, consideramos que ambas as velocidades são válidas e devem ser escolhidas pelo pesquisador.

\section{0 btenção dos resultados quantitativos} e qualitativos

Quando o teste de microtração começou a ser realizado, em 1994, o corpo de prova era a unidade experimental da pesquisa. Com o passar do tempo, a unidade experimental passou a ser o dente que gerava determinado número de corpos de prova.

O valor de resistência de união de cada dente era calculado a partir da média dos valores encontrados por corpo de prova. Todavia, em alguns casos havia um grande número de corpos de prova perdidos devido à fratura prematura, que ocorria principalmente durante o procedimento de corte. Essa perda, porém, não era considerada na análise dos resultados e devido a isso surgiram questionamentos a respeito da validade desses estudos ${ }^{35,36}$.

$\operatorname{Reis}^{75}$ (2002) desenvolveu uma fórmula na qual os palitos perdidos eram levados em conta a fim de se obter a média de resistência de união por grupo experimental:

$$
I_{d}=\frac{\left(R_{A / M} \times n_{A / M}\right)+\left(R_{C} \times n_{C}\right)+\left(R_{p} \times n_{P}\right)}{n_{A / M}+n_{C}+n_{P}}
$$

Onde:

$\mathrm{R}_{\mathrm{A} / \mathrm{M}}$ - média de resistência de união dos corpos de prova com fratura adesiva/ mista;

$\mathrm{n}_{\mathrm{A} / \mathrm{M}}$ - número de corpos de prova com fratura adesiva/mista;

$\mathrm{R}_{\mathrm{C}}$ - média de resistência de união dos
GARBUI BU

AZEVEDO CS

MATOS $A B$

ENSAIO DE

RESISTÊNCIA

ADESIVA POR

MICROTRAÇÃO:

REVISÃO DE

LITERATURA

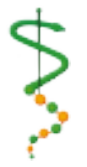

Rev. OdOntOl.

Univ, Cid, São

Paulo

$2013 ; 25(1): 47$

57, JAN-ABR 
GARBUI BU AZEVEDO CS

MATOS $A B$

ENSAIO DE RES ISTENNCIA ADESIVA POR MICROTRAÇÃO: REVISÃO DE LITERATURA

\section{ISSN 1983-5183}

corpos de prova com fratura coesiva de dentina;

$\mathrm{n}_{\mathrm{C}}$ - número de corpos de prova com fratura coesiva de dentina;

$R_{p}$ - valor médio de resistência de união atribuída aos corpos de prova perdidos;

$\mathrm{n}_{\mathrm{p}}$ - número de corpos de prova perdidos

O valor de $R_{p}$ adotado pode ser $0^{18,44}$ ou $4 \mathrm{MPa}^{75}$, sendo este último o valor mínimo aferido no teste de microtração ${ }^{2}$.

Para realizar a análise estatística dos resultados, o teste mais frequentemente utilizado é a Análise de Variância (ANOVA), que geralmente é associado a um teste auxiliar como, por exemplo, o Tukey $^{40,41,43,45,46,59}$ ou o Fisher ${ }^{58}$.

Já para a análise das fraturas, que po- dem ser classificadas em: Adesiva, Coesiva de Substrato, Coesiva de Resina ou Mista, geralmente se usa a observação com Lupa Esteroscópica 9,14,29,31,34,37,38,61,62 ou Microscopia Eletrônica de Varredura $(\mathrm{MEV})^{16,19,23,24,34,41,46,47,58,59}$.

\section{CONCLUSÕES}

Através desta revisão de literatura, foi possível concluir que há muitas metodologias possíveis de serem aplicadas no teste de microtração. Todavia, é necessário ressaltar que os pesquisadores precisam estar treinados e deve haver condições de infraestrutura adequadas para o desenvolvimento da metodologia escolhida pelo grupo de trabalho. 
GARBUI BU AZEVEDO CS MATOS $A B$

ENSAIO DE RESISTÊNCIA ADESIVA POR MICROTRAÇÃO: REVISÃO DE LITERATURA

4. De Munch J, Van Meerbeek B, Satoshi I, Vargas M, Yoshida Y, Armstrong S, et al. Microtensile bond strengths of one- and two-step self etch adhesives to bur-cut enamel and dentin. Am J Dent 2003a; 16(6)414-420.

5. Goracci C, Sadek FT, Monticelli F, Cardoso PEC, Ferrari M. Microtensile bond strength of self-etching adhesives to enamel and dentin. J Adhes Dent 2004a; 6(4):313-318.

6. Garone Filho W. Adesão em esmalte e dentina. In: Cardoso RJA, Gonçalves EAN, editores. Dentística/laser. São Paulo: Artes Médicas; 2000. p. 27-55.

7. Van Meekbeek B, Vargas M, Inoue S, Yoshida Y, Peumans M, Lambrechts P, et al. Adhesives and cements to promote preservation dentistry. Oper Dent 2001; Suppl6:119-144.

8. Pashley EL, Agee KA, Pashley DH, Tay FR. Effects of one versus two applications of an unfilled, all-in-one adhesive on dentine bonding. J Dent 2002;30(2-3):83-90.

9. Sadek FT, Muench A, Poiate IA, Junior EP, Cardoso PEC. Influence of Specimens' Design and Manufacturing Process on Microtensile Bond Strenght to Enamel - Laboratory and FEA Comparison. Materials Research 2010; 13(2):253-260.

10. Anusavice KJ. Materiais de acabamento e polimento. In:materiais dentários. Rio de Janeiro: Guanabara Koogan S.A.; 1998. P. 394-405.

11. Mahoney E, Holt A, Swain M, Kilpatrick N. The hardness and modulus of elasticity of primary molar teeth: an ultra-micro-indentation study. J Dent 2000;28(8):589594.

12. Tay FR, Pashley DH, Yiu CK, Sanares AM, Wei SH. Factors contributing to the incompatibility between simplified-step adhesives and chemically-cured or dual-cured composites. Part I. Single-step self-etching adhesive. J Adhes Dent 2003;5(1):2740.

13. Armstrong SR, Vargas MA, Fang Q, Laffon JE. Microtensile bond strength of a totaletch 3-step, total-etch 2-step, self-etch 2-step, and a self-etch 1-step dentin bonding system through 15-month water storage. J Adhes Dent 2003;5(1):47-56.

14. Belli S, Ozçopur B, Yesilyurt C, Bulut G, Ding X, Sorsman G. The effect of Loading on $\mu$ TBS of Four All-In-One Adhesives On Bonding to Dentin. J. Biomed Mater Res Part B: Appl Biomater 91B:948-956.

15. Chen KK, Shono Y, Ogawa T, Kozono Y, Terashita M. Fractue aspects of resin-dentin bonding in non-trimming microtensile test. Dent Mater J 2001; 20(4):315-324.

16. D’Arcangelo C, Vanini L, Prosperi GD, Bussolo GD, Angelis FD, D'Amario M, Caputi S.

17. Donmez N, Belli S, Pashley D H, Tay F R. Ultrastructural correlates of in vivo/in vitro bond degradation in self-etch adhesives. Journal of Dental Research 2005; 84(4): 355-359. 
GARBUI BU: AZEVEDO CS

MATOS AB

ENSAIO DE RES ISTENNCIA ADESIVA POR MICROTRAÇÃO: REVISÃO DE LITERATURA

REV, ODONTOL. UNIV, CID, SÃO PAULO: $2013 ; 25(1): 47$ 57, JAN-ABR
18. Duarte Jr. S, Phark J, Varjão FM, Sadan A. Nanoleakage, ultramorphological characteristics, and microtensile bend strengths of a new low-shrinjage composite to dentin after artificial agind. Dent Mat 2009; 25: 589-600.

19. Fawzy AS, El-Askary FS, Amer MA. Effect of surface treatments on the tensile bond strength of repaired water-aged anterior restorative micro-fune hybrid resin composite. J Dent 2008; 36:969-976.

20. Gamborgi GP, Loguercio AD, Reis A. Influence of enamel border and regional variability on durability of resin-dentin bonds. Journal of dentistry 2007; 35:371-376.

21. Giannini M, Carvalho RM, Martins LRM, Dias CTS, Pashley DH. The influence of tubule density and area of solid dentin on bond strength of two adhesive systems to dentin. J Adhes Dent 2001;3:315-324.

22. Hashimoto $M$, Ohno $H$, Sano H, Kaga $M$, Oguchi $H$. In vitro degradation of resindentin bonds analyzed by microtensile bond test, scanning and transmission electron microscopy. Biomaterials 2003; 24:3795-3803

23. Hashimoto, M., Fujita, S., Kaga, M., \& Yawaka, Y. In vitro durability of one-bottle resin adhesives bonded to dentin. Dent Mat JI 2007; 26(5): 677-686.

24. Hosaka, K., Nishitani, Y., Tagami, J., Yoshiyama, M., Brackett, W. W., Agee, K. A., et al. Durability of resin-dentin bonds to water- vs. ethanol-saturated dentin. Journal of Dental Research 2009; 88(2): 146-151.

25. Iwasaki K, Miyazaki M, Yoshida Y, Oshida Y. Influence of crosshead speed on micro-tensile bond strength (abstract 331). J Dent Res 2003;82(Spec Iss B):54.

26. Lopes MB, Sinhoreti MAC, Sobrinho LC, Consani S. Comparative study of the dental substrate used in shear bond strength tests. Estudo comparativo do tipo de substrato dental utilizado em testes de resistência de união ao cisalhamento. Pesqui Odontol Bras 2003;17(2):171-5.

27. Louguercio AD, Uceda-Gomez N, Carrilho MR, Reis A. Influence of specimen size and variation on long term resin-dentin strength. Dent Mater 2005; 21:224-231.

28. Meira JB, Souza RM, Driemeier L, Ballester RY. Stress concentration in microtensile test using uniform material. J Adhes Dent 2004; 6(4):267-273.

29. Mobarak EH, El-Badrawy W, Pashley DH, Jamjoom H. Effect of pretest storage conditions of extracted teeth on their dentin bond strengths. The Journal of Prosthetic Dentistry 2010; 104(2):92-97.

30. Monticelli F, Osorio R, Pisani-Proença J, Toledano M. Resistance to degradation of resin-dentin bonds using a one-step HEMA-free adhesive. Journal of Dentistry 2007; 35(2): 181-186.

31. Mountouris G, Silikas N, Eliades G. Effect of sodium hypochlorite treatment on the molecular composition and morphology of human coronal dentin. Journal of Adhesive Dentistry 2004; 6(3): 175-182.

32. Nakabayashi N, Takarada K. Effect of HEMA on bonding to dentin. Dent Mater 1992; 8:125-130.

33. Nikolaenko SA, Lohbauer U, Roggendorf M, Petschelt A, Dasch W, Frankenberger R. Influence of c-factor and layering technique on microtensile bond strength to dentin. Dental Materials 2004; 20:579-585.

34. Özer F, Sengun A, Ozturk B, Say E C, Tagami J. Effect of tooth age on microtensile bond strength of two fluoride-releasing bonding agents. Journal of Adhesive Dentistry 2005; 7(4): 289-295. 
35. Phrukkanon S, Burrow MF, Tyas MJ. Effect of cross-sectional surface area on bond strengths between resin and dentin. Dent Mater 1998a; 14(2):120-128.

36. Phrukkanon S, Burrow MF, Tyas MJ. Th influence of cross-sectional shape and surface area on the microtensile bond test. Dent Mater 1998b; 14(3):212-221.

37. Reis A, de Oliveira Baurer JR, Loguercio AD. Influence of crosshead speed on resindentin microtensile bond strength. J Adhesiv Dent 2004;6:275-278.

38. Reis A, Zander-Grande C, Kossatz S, Stanislawczuk R, Manso A, Carvalho RM, Loguercio AD. Effect of mode of application on the microtensile bond strength of a self-etch and etch-and-rinse adhesive system. Operative Dentistry 2010; 35 (4): 428-435.

39. Reis AF, Bedran-Russo AKB, Giannini M, Pereira PNR. Interfacial ultramorphology of single-step adhesives: Nanoleakage as a function of time. Journal of Oral Rehabilitation 2007; 34(3): 213-221.

40. Reis AF, Giannini M, Pereira PNR. Long-term TEM analysis of the nanoleakage patterns in resin-dentin interfaces produced by different bonding strategies. Dental Materials 2007; 23(9):1164-1172.

41. Rocha R, Soares F Z M, Rodrigues C R M D, Rodrigues Filho L E. Influence of aging treatments on microtensile bond strength of adhesive systems to primary dentin. Journal of Dentistry for Children 2007; 74(2): 109-112.

42. Saboia VP, Nato F, Mazzoni A, Orsini G, Putignano A, Giannini M, et al. Adhesion of a two-step etch-and-rinse adhesive on collagen-depleted dentin. The Journal of Adhesive Dentistry 2008; 10(6): 419-422.

43. Sadek F T, Castellan CS, Braga RR, Mai S, Tjäderhane L, Pashley DH, et al. One-year stability of resin-dentin bonds created with a hydrophobic ethanol-wet bonding technique. Dental Materials 2010; 26(4): 380-386.

44. Sadek FT, Cury AH, Monticelli F, Ferrari M, Cardoso PEC. The influence of the cutting speed on bond strength and integrity of microtensile specimens. Dent Mater. 2005; $21: 1-6$.

45. Sarr M, Kane AW, Vreven J, Mine A, Van Landuyt KJ, Peumans M, Lambrechts P, Van Meerbeek B, De Munck J. Microtensile bond strength and interfacial characterization of 11 contemporary adhesives bonded to bur-cut dentin. Operative Dentistry 2010;35 (1): 94-104.

46. Sattabanasuk V, Vachiramon V, Qian F, Armstrong SR. Resin-dentin bond strength as related to different surface preparation methods. Journal of Dentistry 2007; 35(6):467-475.

47. Semeraro S, Mezzanzanica D, Spreafico D, Gagliani M, Re D, Tanaka T, Sidhu SK, Sano $\mathrm{H}$. Effect of different bur grinding on the bond strength of self-etching adhesives Operative Dentistry 2006; 31 (3): 317-323.

48. Sevgican, F., Inoue, S., Koase, K., Kawamoto, C., Ikeda, T., Sano, H. Bond strength of simplified-step adhesives to enamel prepared with two different diamond burs. Australian Dental Journal 2004; 49(3):141-145.

49. Shinkai K, Ebihara T, Shirono M, Seki H, Wakaki S, Suzuki M, Suzuki S, Katoh Y. Effects of attrition, prior acid-etching, and cyclic loading on the bond strength of a self-etching adhesive system to dentin. Dental Materials Journal 2009; 28(2):197203/

50. Shinohara MS, Goes MFD, Schneider LFJ, Ferracane JL, Pereira PNR, Hipólito VD, Nikaido T. Dental Materials 2009; 25:1383-1391.

GARBUI BU AZEVEDO CS MATOS AB

ENSAIO DE RESISTENNCIA ADESIVA POR MICROTRAÇÃO: REVISÃo DE LITERATURA

\section{5}

ReV. OdOnTOL. Univ, Cid, São Paulo 2013; 25(1): 47 57, JAN-ABR 
GARBUI BU: AZEVEDO CS MATOS $A B$

ENSAIO DE RES ISTÊNCIA ADESIVA POR MICROTRAÇÃO: REVISÃO DE LITERATURA

REV, ODONTOL, UNIV, CID, SÃO PaUlo $2013 ; 25(1): 47$ $57, J A N-A B R$
51. Shono Y, Ogawa T, Terashita M, Carvakho RM, Pashley EM, Pashley DH. Regional measurement of resin-dentin bonding as an array. J Dent Res 1999; 78(2):699-705.

52. Shono Y, Terashita M, Pashley EL, Brewer PD, Pashleu DH. Effects of cross-sectional area on resin-enamel tensile bond strength. Dent Mater 1997; 13(5):290-296.

53. Tao L, Pashely DH, Boyd L. Effect of different types of smear layers on dentin and enamel shear bond strengths. Dent Mater 1988;4(4):208-16.

54. Tay FR, Hashimoto M, Pashley DH, Peters MC, Lai SCN, Yiu CKY, et al. Aging affects two modes of nanoleakage expression in bonded dentin. Journal of Dental Research 2003; 82(7): 537-541.

55. Tay FR, Pashley DH, Yoshiyama M. Two Modes of Nanoleakage Expression in Single- step Adhesives. J Dent Res 2002; 81(7):472-476.

56. Tosun G, Sener Y, Sengun A. Effect of storage duration/solution on microshear bond strength of composite to enamel. Dent Mater J 2007; 26:116-121.

57. Yamaguchi K, Miyazaki M, Takimizawa T, Tsubota K, Rikta A. Influence of crosshead speed on micro-tensile bond strength of two-step adhesive systems. Dent Mater 2006;22:420-425.

58. Yamauti M, Hashimoto M, Sano H, Ohno H, Carvalho RM, Kaga M, et al. Degradation of resin-dentin bonds using $\mathrm{NaOCl}$ storage. Dental Materials 2003; 19(5):399405.

59. Yoshida E, Hashimoto M, Hori M, Kaga M, SanoH, Oguchi H. Deproteinizing Effects on Resin Tooth Bond Structures. J Biomed Mater 2003;68B:29-35.

60. Yoshiyama M, Carvalh RM, Sano H, Horner JA, Brewer PD, Pashley DH. Regional bong strengths of resins to human root dentine. J Dent 1996; 24(6):435-442.

61. Zohairy AAE, Saber MH, Abdalla AI, Feilzer AJ. Efficacy of microtensile versus microshear bond testing for evaluation of bond strength of dental adhesive systems to enamel. Dental Materials 2010; 26:848-854.

62. Betamar N, Cardew G, Noort RV. Influence of Specimen Designs on the Microtensile Bond Strength to Dentin. The Journal of Adhesive Dentistry 2007; 9(2):159-168.

63. Dias WRL, Pereira PNR, Swift Jr EJ. Effect of Surface Preparation on Microtensile Bond Strenght of Three Adhesive Systems to Bovine Enamel. J Adhesive Dentistry 2004; 6(2): 279-285.

64. Lopes MB. Análise estrutural e mecânica de dentes bovinos relacionados a testes de união adesiva. Piracicaba 2007: 15-40. Tese (Doutorado). Faculdade de Odontologia da Universidade da Campinas (UNICAMP).

65. Nakamichi I, Iwaku M, Fusayama T. Bovine teeth as possible substitutes in the adhesion test. J Dent Res. 1983 Oct;62(10):1076-81.

66. Poitevin A, De Munch J, Van Laduyt K, Coutinho E, Peumans M, Lambrechts P, et al. Critical analysis of the influence of different parameters on the microtensile bond strength of adhesives to dentin. J Adhesiv Dent 2008;10:7-16.

67. Reis AF, Giannini M, Kavaguchi A, Soares CJ, Line SRP. Comparison of microtensile bond strength to enamel and dentin of human, bovine, and porcine teeth. J Adhesive Dentistry 2004; 6(2):117-121.

68. Titley KC, Chernecky R, Rossouw PE, Kulkarni GV. The effect of various storage methods and media on shear-bond strengths of dental composite resin to bovine dentine. Arch Oral Biol 1998; 43:305-311. 
69. Tonami K, Takahashi H, Nishimura F. Effect of frozen storage and boiling on tensile strenght of bovine dentin. Dent Mater J 1996; 15:205-211.

70. Uekusa S, Tsubota K, Tonegawa M, Tsuchiya H, Iwasa M, Kawamoto R, Takamizawa T, Miyazaki M. Microtensile bond strengths of single-step self-etch adhesive systems to bovine dentin. J Oral Science 2007; 49(3):183-189.

71. Pashley DH, Sano H, Ciucchi B, Yoshiyama M, Carvalho RM. Adhesion testing of dentin bonding agents: a review. Dental Materials 1995; 11(2):117-125.

72. Saam T, Kerwin WS, Chu B, Cai J, Kampschulte A, Hatsukami TS, et al. Sample size calculation for clinical trials using magnetic resonance imaging for the quantitative assessment of carotid atherosclerosis. J Cardiovasc Magn Reson. 2005;7: 799-808.

73. Ferreira MC. Preparo de amostras para a realização do teste de microdureza. 2004, 32p. http://www.ccs.ufsc.br/posodonto/Preparoamostras_microdureza.pdf> Acesso em: 30 out. 2007.

74. Oliveira SS, Pugach MK, Hilton JF, Watanabe LG, Marshall SJ, Marshall Jr GW. The influence of the dentin smear layer on adhesion: a self-etching primer vs. a totaletch system. Dent Mat 2003; 19: 758-767.

75. Reis AS. Espectro da umidade da superfície dentinária para três sistemas adesivos com diferentes solventes. São Paulo 2002: 44-48. Tese (Doutorado). Faculdade de Odontologia da Universidade de São Paulo (FOUSP).

Recebido em: 10/11/2011

Aceito em: 28/11/2011
GARBUI BU AZEVEDO CS

MATOS AB

ENSAIO DE RESISTENNCIA ADESIVA POR MICROTRAÇÃO: REVISÃO DE LITERATURA 